\title{
Internación compulsiva de usuarios de crack para el tratamiento de la dependencia: una revisión sistemática de la literatura
}

\author{
*Aldo Pacheco Ferreira ${ }^{1}$, Priscila Marcia Costa Assumpção Silva ${ }^{1}$, Marluce Rodrigues Godinho ${ }^{2}$, Regina \\ Maria de Carvalho Erthal ${ }^{1}$, Maria Helena Barros de Oliveira ${ }^{1}$, Marcos Besserman Vianna ${ }^{1}$ \\ ${ }^{1}$ Fundação Oswaldo Cruz, Escola Nacional de Saúde Pública Sergio Arouca, Departamento de Direitos Humanos, \\ Saúde e Diversidade Cultural. Rio de Janeiro. Brasil \\ ${ }^{2}$ Universidade Federal de Juiz de Fora, Departamento de Enfermagem Básica (EBA) da Faculdade de Enfermagem. \\ Juiz de Fora, Minas Gerais, Brasil
}

Cómo referenciar este artículo/ How to reference this article:
Pacheco Ferreira A, Costa Assumpção Silva PM, Rodríguez Godinho M, de Carvalho Erthal RM, Barros de Oliveira MH, Besserman Vianna M. Internación compulsiva de usuarios de crack para el tratamiento de la dependencia: una revisión sistemática de la literatura. Mem. Inst. Investig. Cienc. Salud. 2019; 17(1):113-124

\section{RES U M E N}

El estudio tuvo como objetivo discutir los conflictos de la internación compulsiva de consumidores de crack. Se realizó una revisión sistemática sobre el tratamiento compulsivo. La búsqueda fue realizada en las bases de datos: Scopus, PubMed, SciELO, Web of Science, Elsevier y Medline, así como en Google y Google Scholar, y se incluyeron publicaciones de 2000 a 2017. El desenlace primario de interés fue el uso de drogas post-tratamiento, y el secundario la reincidencia criminal pos-tratamiento. De un total de 448 estudios potenciales identificados, doce estudios cumplieron los criterios de inclusión. Se concluye que la internación compulsiva, además de ser agresiva y una forma de tratamiento ineficaz, constituye un modo de eliminación de los indeseados, constituyéndose en práctica higienista violadora de derechos humanos.

Palabras clave: cocaína crack, conducta adictiva, toxicología forense, derechos humanos, política de salud, control social, salud pública.

\section{Compulsory commitment of crack users for addiction treatment: A systematic review of the literature}

\begin{abstract}
A B S T R A C T
The study aimed to discuss the conflicts of compulsory commitment of crack users. A systematic review was conducted on compulsory treatment. The search was conducted in databases: Scopus, PubMed, SciELO, Web of Science, Elsevier and Medline, as well as in Google and Google Scholar, from 2000 to 2017. The primary endpoint of interest was the use of post-treatment drugs. The secondary outcome of interest was post-treatment criminal recidivism. Of a total of 448 potential studies identified, twelve studies met the inclusion criteria. It was denoted that compulsory hospitalization, besides being aggressive and an ineffective treatment, constitutes a way of eliminating the unwanted, constituting a hygienist practice that violates human rights.
\end{abstract}

Keywords: crack cocaine, addictive behavior, forensic toxicology, human rights, health policy, social control, public health. 


\section{INTRODUCCIÓN}

El crack es una droga ilegal consumida con alta frecuencia y con graves consecuencias orgánicas, psiquiátricas y sociales. Aunque cada vez los datos disponibles son mayores, es probable que el abordaje del problema aún diste de ser el adecuado dada la frecuencia del consumo y de sus complicaciones ${ }^{(1)}$. Los usuarios tienen mucha dificultad en la búsqueda de tratamiento especializado, pues no reconocen el problema, enfrentan prejuicio por la ilegalidad de la droga ligada a la criminalidad, el acceso al tratamiento es difícil y los servicios especializados no ofrecen la intervención ajustada a sus necesidades ${ }^{(2)}$.

Mientras que la cocaína en polvo (clorhidrato) se ha utilizado durante décadas, el crack surgió como un subtipo en la década de $1980^{(3)}$. En Brasil, los servicios ambulatorios especializados para tratar la dependencia del uso de crack comenzaron a sentir el impacto del crecimiento del consumo a partir de principios de los años 1990, cuando en algunos centros la proporción de usuarios fue del $17 \%$ (1990) para el $64 \%(1994)^{(4)}$. Las complicaciones relacionadas con el consumo de Crack capaces de llevar al individuo a la atención médica son habitualmente agudas e individuales ${ }^{(5)}$.

La dependencia de Crack viene caracterizándose como un problema de salud pública, no tanto por la frecuencia del uso, sino por la intensidad de la dependencia y los daños para la salud resultantes ${ }^{(6)}$. En virtud de estos aspectos, la dependencia de crack se ha convertido en una de las importantes demandas en ambientes ambulatorios y hospitalarios de tratamiento ${ }^{(5,7)}$.

A pesar de la implementación generalizada de modalidades de tratamiento compulsivo para la adicción a las drogas, no existe una evaluación de las evidencias científicas sobre la eficacia de este tratamiento. Por lo tanto cabe destacar que, a nivel mundial, la dependencia de drogas ilícitas y no registradas sigue siendo una fuente clave de morbosidad y mortalidad, y está implicada con el mundo del crimen.

Por consiguiente, la intención es llamar la atención sobre la interpretación judicial que viene siendo aplicada conforme a la Ley Federal no $10.216 / 01^{(8)}$, que dispone sobre la protección y los derechos de las personas portadoras de trastornos mentales y reapunta el tipo asistencial en salud mental en Brasil, la red de atención sustitutiva aún no ha sido suficiente para garantizar la reestructuración y el cuidado en salud mental en general y en particular a los consumidores de crack en su plenitud.

Esta Ley es el resultado del proceso de luchas y marco legal de la Reforma Psiquiátrica Brasileña. Incluso tras debates y avances en el campo de la salud mental, los medios permeados por sus relaciones políticas y comerciales vienen retomando discursos higienistas ante estos individuos. Esto es visto por el modo asustador como aborda el asunto en sus reportajes y noticiario, destacando la limpieza social, la internación compulsiva y contenidos regados por denominaciones peyorativas. Esta práctica legitima repulsa de la sociedad ante los consumidores.

Aunque la Ley existe desde 2001, fueron las ocurrencias de internación compulsiva hecha por las alcaldías de los municipios de Río de Janeiro y São Paulo, ya sea a petición de las familias de consumidores de crack o de las propias prefecturas, que la puso en evidencia. Paralelamente, demuestra por medio de análisis sociopolítico, que la internación compulsiva no puede ser asumida como la medida que sanará el grave problema de dependencia química tan fuertemente instalado en nuestra sociedad. Se debe aclarar que se tiene como premisa básica que el dependiente químico no es enfermo mental ${ }^{(9)}$.

Se estima que 18,3 millones de personas entre 16 y 64 años consumieron cocaína en 2014, lo que representa el $0,3-0,4 \%$ de la población mundial, convirtiendo a la cocaína en una de las drogas ilícitas más utilizadas ${ }^{(10)}$. En 2013 , la propagación estimada del trastorno por consumo de cocaína fue de 7,4 millones de casos, lo que representa 1,0 millones de años vividos con discapacidad, a nivel mundial ${ }^{(11)}$. La internación compulsiva, en los moldes que viene siendo requerida, especialmente por órganos del Poder Ejecutivo, y ratificada por el Poder Judicial, transgrede frontalmente los principios constitucionales de la dignidad de la persona humana y de la salud, que también se consideran especies de derechos humanos y fundamentales ${ }^{(12,13)}$. En muchos ajustes, las modalidades de tratamiento compulsorio se han implementado o se están implementando. Por ejemplo, una revisión internacional reciente encontró que a partir de 2009 , el $69 \%$ de una muestra de países $(n=104)$ tenía leyes criminales que permitían el tratamiento compulsorio de $\operatorname{drogas}^{(14)}$. 
El tratamiento compulsorio con medicamentos (particularmente en pacientes hospitalizados) a menudo se basa en la abstinencia, y generalmente está anidado dentro de una respuesta más amplia orientada a la justicia penal para los daños relacionados con las drogas ${ }^{(15)}$. El tratamiento compulsorio es distinto del tratamiento forzado, por el hecho de que se trata de tratar la dependencia a las drogas por medio de cuidados a la salud y no del castigo ${ }^{(16,17)}$. Quizás el ejemplo más ampliamente conocido de tratamiento forzado es el modelo de tribunal de tratamiento de drogas, que proporciona a las personas acusadas de un delito relacionado con drogas medidas terapéuticas además de intervenciones de justicia penal bajo los auspicios del sistema de justicia penal ${ }^{(18)}$.

Si bien no se realizó una evaluación sistemática de la efectividad de los enfoques de internación compulsiva, los observadores mencionaron preocupaciones sobre violaciones de derechos humanos dentro de los centros de tratamiento de drogas ${ }^{(19,20)}$. Además, aunque existen panoramas, así como revisiones sobre temas relacionados $(21,22)$, no se han realizado evaluaciones sistemáticas recientes de la eficacia o efectividad de programas tratamientos compulsorios o forzados. Esto representa una brecha crítica en la literatura dada la implementación y ampliación del tratamiento compulsorio en una variedad de ajustes.

De una forma generalizada, los consumidores de crack han sido causantes de una histeria social y la construcción de estigmas, que son la parte visible para la sociedad del consumo de drogas, justamente la parte de este comportamiento que no es tolerado por ella ${ }^{(23,24)}$. Según Arenari y Dutra ${ }^{(25)}$ el presupuesto central se debe al hecho de que uno de los problemas sociales más frecuentes es la exclusión social. Comúnmente es demostrado por los medios llevando a la formación de un tipo de identidad marcada por la participación precaria en la mayoría del ámbito social y en el linde a la negación del status de persona a los individuos.

Entonces, considerando que la imagen del consumidor de crack no resulta necesariamente de sí mismo, pero es producto de los que lo ve o lo ignoran, y considerando también que es fruto de una variedad de instituciones que los interpreta, entre ellos los medios, movida por la ideología de la clase dominante, hace sentido que estos medios les atribuyan expectativas de conducta específicas que terminan confirmando el sentido de exclusión preexistente, como si fuera un ciclo $^{(13)}$.

Se resalta que el objeto de este estudio es el consumidor de drogas mayor de 18 años, que tiene su internación requerida judicialmente. Sin embargo, todas las ponderaciones aquí realizadas pueden ser utilizadas como un argumento contrario a la internación obligatoria de menores de 18 años. El hecho de que la minoría no les otorgue capacidad civil, por sí sola, no es bastante para que no hayan afianzado su derecho a la salud.

Con efecto, en cualquier argumento que se vea la cuestión, la conclusión es siempre la misma: la internación compulsiva de dependientes químicos, mayores o menores de edad, no les garantiza el derecho a la vida, al contrario, constituye violación de su derecho a la salud. Así, dada la necesidad de evidencia científica para fundamentar enfoques efectivos del tratamiento de drogas, llevamos a cabo una revisión sistemática de las acciones que involucran el encarcelamiento compulsorio de usuarios crack.

\section{MATERIALES Y MÉTODOS}

Empleamos el Preferred Reporting Items for Systematic Reviews and Meta-Analyses (PRISMA) directrices para el desarrollo de la revisión sistemática ${ }^{(26)}$.

\section{Criterio de elegibilidad}

Los estudios fueran elegibles si revisados por pares, y si evaluaban el impacto del encarcelamiento compulsorio sobre los resultados relacionados con crack. El resultado primario de interés se definió como la frecuencia del uso de la droga después del tratamiento. El resultado secundario de interés se definió como cualquier reincidencia criminal relacionada con el medicamento posterior al tratamiento (es decir, arresto o encarcelamiento posterior al tratamiento). Los ensayos clínicos controlados aleatorizados y los estudios observacionales fueron elegibles para la inclusión. Para ser elegible, las intervenciones de tratamiento informadas debían ser compulsivas; sin embargo, el tipo de intervención (por ejemplo, terapia basada en la abstinencia para pacientes hospitalizados, terapia grupal ambulatoria, etc.) podría variar. Las revisiones y los estudios multicomponente que no se desagregaron los hallazgos entre los componentes no fueron elegibles si no proporcionaban datos específicos 
sobre el impacto del encarcelamiento compulsorio. Los estudios que evaluaron el encarcelamiento compulsorio para sustancias legales o lícitas (es decir, alcohol, tabaco) tampoco fueron elegibles. Además, se excluyeron los estudios que solo evaluaron los resultados, como el cambio de actitud o psicosocial, o el funcionamiento psicológico relacionado con el uso de sustancias. Finalmente, se excluyeron los estudios que evaluaron el tratamiento forzado o cuasi-compulsivo (es decir, en el que los individuos tienen la opción de elegir entre el tratamiento y un resultado punitivo como el encarcelamiento, como un modelo de tribunal de tratamiento de drogas).

\section{Fuentes de información}

Se realizó búsqueda en bases electrónicas de publicaciones científicas, siendo identificadas las siguientes: Scopus; Public/Publisher Medline (PubMed); Scientific Electronic Library Online (SciELO), Web of Science; Elsevier y Medical Literature Analysis and Retrieval System Online (Medline). También se buscó en Internet (Google, Google Scholar), listas de resúmenes de conferencias académicas relevantes y escaneamos las referencias de estudios potencialmente elegibles.

\section{Estrategia de búsqueda}

Los términos Medical Subject Headings (MESH) investigados fueron: "forced treatment," "compulsory treatment," "substance abuse," "substance use," "mandated treatment," "mandatory treatment," "addiction," "addiction treatment," "involuntary treatment," "involuntary addiction treatment." El periodo de recogida de artículos fue de enero 2000 a diciembre 2017 en los idiomas inglés, castellano y portugués.

\section{Selección de estudios}

Para la definición de los artículos seleccionados, fueron hechas la lectura de los resúmenes y de los artículos completos. Fueran escaneados todos los resúmenes y se obtuvo textos completos de los artículos que potencialmente cumplían con los criterios de elegibilidad.

Se aplicó el protocolo AMSTAR (Assessment of Multiple Systematic Reviews) ${ }^{(27)}$ con un alcance de 9 ítems dentro de los 11 solicitados (sólo los ítems 'no realización de prueba de homogeneidad' y 'no acceso a la literatura gris' no se aplicaron a ese tipo de revisión).

La síntesis del resultado de la búsqueda realizada en las bases investigadas se encuentra sistematizada en el diagrama de flujo (Figura 1).

\section{Evaluación de la calidad metodológica}

La evaluación sistemática de la calidad fue hecha por la puntuación de Downs y Black (28). Esta herramienta objetiva la evaluación de la calidad metodológica y fue especialmente diseñada para contemplar estudios aleatorizados y no aleatorizados, y presenta 27 ítems puntuables. 


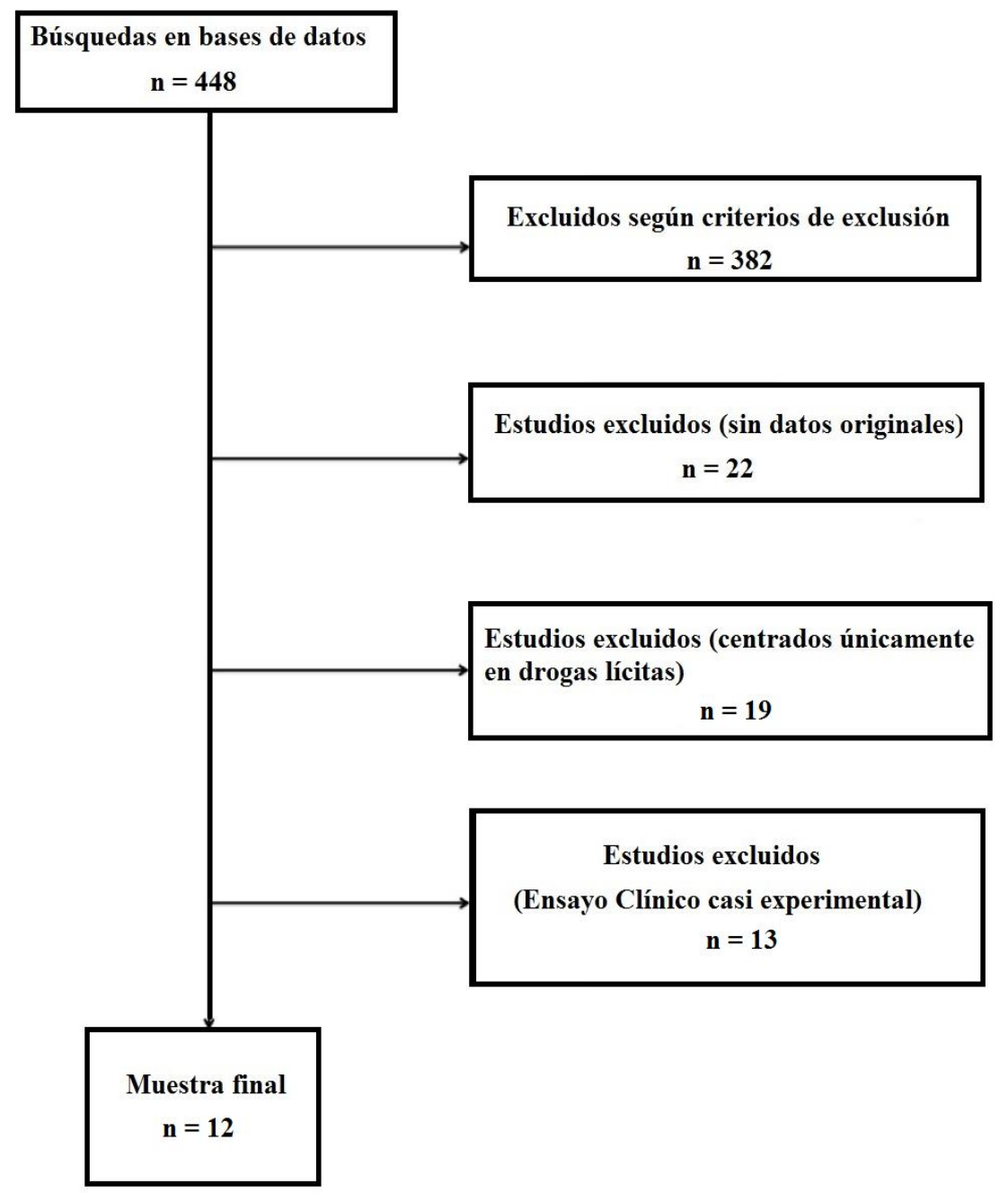

Figura 1: Estrategia de búsqueda de artículos para la revisión sistemática que evaluaba el efecto de la internación compulsiva de consumidores de crack: 2000-2017

\section{RESULTADOS}

\section{Selección de estudios y características}

Se identificaron inicialmente 448 estudios, de los cuales 382 se excluyeron porque no presentaron datos primarios y/o específicos sobre el tratamiento compulsorio. De los 66 estudios restantes, 22 se excluyeron porque constituían revisiones o editoriales, 19 se excluyeron porque no se centraron en el uso de drogas ilícitas (es decir, se centraron en el tratamiento del alcoholismo) y se excluyeron 13 estudios porque evaluaron el tratamiento cuasi activo en lugar de intervenciones de tratamiento compulsorio. 12 estudios cumplieron los criterios de inclusión. Cinco estudios emplearon abordajes observacionales longitudinales, cuatro estudios emplearon diseños prospectivos de control de casos, dos estudios emplearan un diseño de corte transversal y un estudio empleó un diseño cuasi-experimental. Seis estudios evaluaron el tratamiento hospitalario compulsorio o la detención de drogas, un estudio evaluó el tratamiento en prisión/detención, y dos estudios evaluaron el tratamiento comunitario compulsorio.

\section{Análisis de la muestra final}

La distribución temporal de las 12 publicaciones analizadas fue la siguiente: 2 artículos (2001, 2017); 1 artículo (2003, 2005, 2006, 2009, 2010, 2011, 2014, 2015), respectivamente.

Cinco estudios encontraron resultados positivos: un estudio apoya el uso de intervenciones multisectoriales amplias, integrando promoción de la salud y rehabilitación de la salud mental, acceso a la educación de calidad y gestión del uso combinado/concomitante de diferentes 
sustancias para reducir y/o prevenir la implicación criminal de individuos que usan crack y otras sustancias, así como para prevenir la recaída ${ }^{(29)}$; un estudio observó que la internación obligatoria tiene importantes consecuencias para la sociedad ${ }^{(30)}$; un estudio observó que los usuarios en tratamiento parecían ser más integrados socioeconómicamente y conectados al sistema de salud, pero no agudamente más necesitados en términos de problemas de salud o de drogas ${ }^{(31)}$; un estudio observó un pequeño impacto significativo del tratamiento de internación compulsiva en la reincidencia criminal ${ }^{(32)}$ y un estudio retrospectivo descubrió mejores resultados de uso de drogas en la primera semana después del tratamiento( ${ }^{(33)}$. Tres estudios no reportaron ningún impacto significativo del tratamiento obligatorio sobre el uso de sustancias en comparación con las intervenciones de control ${ }^{(34-36)}$. Dos estudios encontraron resultados ambiguos, pero no se compararon con una condición de control (por ejemplo, tratamiento medicamentoso voluntario) ${ }^{(33,37)}$. Dos estudios observaron impactos negativos del tratamiento compulsivo en la reincidencia criminal ${ }^{(38,39)}$ (Cuadro 1 ).

Cuadro 1. Resultados de la revisión sistemática de los estudios que evidenciaron la internación compulsiva de usuarios de crack

\begin{tabular}{|c|c|c|c|c|}
\hline Año & Autor(es) & Publicación & Diseño & Resultado \\
\hline 2017 & Heather & Addictive Behaviors Reports & Descriptivo & $\begin{array}{l}\text { El comportamiento adictivo } \\
\text { no puede considerarse } \\
\text { compulsivo en el momento } \\
\text { en que se lleva a cabo, } \\
\text { aunque se discuten otros } \\
\text { posibles significados de } \\
\text { compulsión como una } \\
\text { explicación o descripción del } \\
\text { comportamiento adictivo y la } \\
\text { experiencia. Se sugiere que, } \\
\text { aunque en algunos sentidos } \\
\text { del término puede parecer } \\
\text { arbitrario si debe conservarse } \\
\text { o no la "compulsión", su uso } \\
\text { tiene importantes } \\
\text { consecuencias para la } \\
\text { comprensión pública de la } \\
\text { adicción y es probable que } \\
\text { disuada los intentos de las } \\
\text { personas de superar sus } \\
\text { adicciones y sus posibilidades } \\
\text { de éxito. }\end{array}$ \\
\hline 2017 & Toledo et al. & $\begin{array}{l}\text { International Journal of Drug } \\
\text { Policy }\end{array}$ & $\begin{array}{l}\text { Muestreo } \\
\text { probabilístico } \\
\text { conglomerado }\end{array}$ & $\begin{array}{l}\text { La mayoría de los } \\
\text { consumidores de crack } \\
\text { reclutados fueron varones } \\
(78.2 \%), 18-30 \text { años } \\
(64.7 \%]) \text {, no-blancos } \\
(92.9 \%) \text {, solo }(68.9 \%) \text {, y con } \\
0-7 \text { años de escolaridad } \\
(70.6 \%) \text {. En cuanto a la } \\
\text { historia de vida de la variable } \\
\text { de resultado del } \\
\text { encarcelamiento, los factores } \\
\text { independientes asociados } \\
\text { fueron: sexo masculino y uso } \\
\text { prolongado de crack y } \\
\text { sustancias relacionadas. }\end{array}$ \\
\hline 2015 & $\begin{array}{l}\text { Fairbairn, } \\
\text { Hayashi, y Ti }\end{array}$ & Drug and Alcohol Review & $\begin{array}{l}\text { Transversal } \\
\text { observacional }\end{array}$ & $\begin{array}{l}\text { Entre } 422 \text { participantes, } 209 \\
(49.5 \%) \text { informaron un } \\
\text { período de cese del uso de } \\
\text { drogas de al menos un año. } \\
\text { La detención compulsiva de } \\
\text { drogas no está asociada con } \\
\text { el cese a largo plazo. El cese } \\
\text { del consumo de drogas se } \\
\text { debió con mayor frecuencia al } \\
\text { encarcelamiento ( } 74 \%), \text { y la } \\
\text { recaída se asoció con la }\end{array}$ \\
\hline
\end{tabular}




\begin{tabular}{|c|c|c|c|c|}
\hline & & & & $\begin{array}{l}\text { liberación de la prisión } \\
(66 \%) \text {. }\end{array}$ \\
\hline 2014 & Cruz et al. & $\begin{array}{l}\text { Substance Abuse Treatment, } \\
\text { Prevention, and Policy }\end{array}$ & $\begin{array}{l}\text { Muestras de } \\
\text { conveniencia }\end{array}$ & $\begin{array}{l}\text { La edad promedio en ambas } \\
\text { muestras fue de } 21 \text { años. Las } \\
\text { mayorías respectivas en } \\
\text { ambas muestras fueron } \\
\text { masculinas; solo o separado; } \\
\text { desempleados y arrestados; } \\
\text { una minoría en ambos grupos } \\
\text { dedicados al trabajo sexual. } \\
\text { Ambas muestras, en } \\
\text { promedio, tenían un historial } \\
\text { de aproximadamente } 4 \text { años } \\
\text { de uso de crack y reportaron } \\
\text { entre } 10 \text { y } 12 \text { episodios de } \\
\text { uso de crack por día. Una } \\
\text { mayor proporción de } \\
\text { participantes sin tratamiento } \\
\text { compartió implementos de } \\
\text { tubería de crack. } \\
\text { Aproximadamente la mitad o } \\
\text { más participantes en ambas } \\
\text { muestras informaron sobre el } \\
\text { uso de alcohol, tabaco, } \\
\text { marihuana y cocaína durante } \\
\text { toda la vida. }\end{array}$ \\
\hline 2011 & $\begin{array}{l}\text { Huang, Zhang } \\
\text { y Liu }\end{array}$ & $\begin{array}{l}\text { International Journal of Drug } \\
\text { Policy }\end{array}$ & $\begin{array}{l}\text { Longitudinal } \\
\text { observacional }\end{array}$ & $\begin{array}{l}46 \% \text { de los encuestados } \\
\text { informaron haber usado } \\
\text { drogas ilícitas dentro de un } \\
\text { mes a seis meses después de } \\
\text { la liberación del tratamiento } \\
\text { obligatorio; otro } 10 \% \text { recayó } \\
\text { dentro de un año. }\end{array}$ \\
\hline 2010 & $\begin{array}{l}\text { Rengifo y } \\
\text { Stemen }\end{array}$ & Crime \& Delinquency & Caso y control & $\begin{array}{l}\text { Impacto no significativo en la } \\
\text { reincidencia en comparación } \\
\text { con las correcciones de la } \\
\text { comunidad; aumento en } \\
\text { comparación con servicios } \\
\text { judiciales. }\end{array}$ \\
\hline 2009 & $\begin{array}{l}\text { Jansson, Hesse } \\
\text { y Fridell }\end{array}$ & $\begin{array}{l}\text { European Addiction } \\
\text { Research }\end{array}$ & $\begin{array}{l}\text { Longitudinal } \\
\text { observacional }\end{array}$ & $\begin{array}{l}\text { La reincidencia se asoció con } \\
\text { el uso de opiáceos. }\end{array}$ \\
\hline 2006 & $\begin{array}{l}\text { Hiller, Knight y } \\
\text { Simpson }\end{array}$ & The Prison Journal & $\begin{array}{l}\text { Longitudinal } \\
\text { observacional }\end{array}$ & $\begin{array}{l}\text { Los graduados en tratamiento } \\
\text { son menos propensos a ser } \\
\text { arrestados dentro de los } 2 \\
\text { años posteriores a dejar el } \\
\text { programa. }\end{array}$ \\
\hline 2005 & $\begin{array}{l}\text { Kelly, Finney y } \\
\text { Moos }\end{array}$ & $\begin{array}{l}\text { Journal of Substance Abuse } \\
\text { Treatment }\end{array}$ & Caso y control & $\begin{array}{l}\text { Los pacientes obligatorios } \\
\text { tenían un perfil clínico menos } \\
\text { severo en la ingesta de } \\
\text { tratamiento; no hay } \\
\text { diferencias en las ganancias } \\
\text { terapéuticas durante el } \\
\text { tratamiento. }\end{array}$ \\
\hline 2003 & $\begin{array}{l}\text { Vaughn, Deng } \\
\text { y Lee }\end{array}$ & Journal of Drug Issues & $\begin{array}{l}\text { Cuasi- } \\
\text { experimental }\end{array}$ & $\begin{array}{l}\text { El grupo de tratamiento tuvo } \\
\text { peores resultados que el } \\
\text { grupo sin tratamiento. }\end{array}$ \\
\hline 2001 & Sun, Ye y Qin & $\begin{array}{l}\text { Chinese Journal of Drug } \\
\text { Dependence }\end{array}$ & Transversal & $\begin{array}{l}\text { Casi todos los participantes } \\
\text { recayeron dentro de un año. } \\
\text { Sin diferencia significativa } \\
\text { entre los participantes } \\
\text { inscritos en diferentes } \\
\text { intervenciones }\end{array}$ \\
\hline
\end{tabular}

\section{DISCUSIÓN}

Siete estudios evaluaron el tratamiento compulsorio con hospitalización o detención de drogas ${ }^{(25,32,34-37,39)}$. Huang et al. ${ }^{(39)}$ examinaron el impacto del tratamiento de pacientes con internación compulsiva en los patrones de uso de drogas post-tratamiento durante el período de un año entre los participantes en Chongquing, China $(n=177)$. Como señalan los autores, la policía china tiene autoridad sobre las instalaciones de tratamiento de drogas y el poder de 
detener individuos dentro de estas instalaciones por un período de semanas a varios meses. Si bien la asignación del tratamiento varía según el centro, las modalidades de tratamiento comúnmente ofrecidas incluyen "ejercicio físico, educación moral y legal, educación sobre drogas y salud, y capacitación en habilidades (por ejemplo, habilidades informáticas)". Sin embargo, los autores no proporcionan datos específicos sobre el contenido de ninguna de estas actividades. Tampoco especificaron el tipo de tratamiento que recibieron los participantes, refiriéndose solo al tratamiento y al asesoramiento. No obstante, $46 \%$ de los encuestados informaron haber usado drogas ilícitas dentro de un mes a seis meses después de la finalización del tratamiento; otro $10 \%$ recayó dentro de un año.

Dos estudios evaluaron el tratamiento compulsorio en ambulatorio o comunitario con una duración de seis meses. Strauss y Falkin ${ }^{(33)}$ trataron de determinar el impacto a corto plazo de una intervención de tratamiento compulsorio comunitario en una muestra de mujeres delincuentes que usaban drogas (crack) en Portland, Oregón $(n=165)$. En un análisis retrospectivo centrado en la primera semana después de la liberación del tratamiento, los autores encontraron que las mujeres que estaban en tratamiento por más tiempo tenían menos probabilidades de consumir drogas durante la primera semana. Rengifo y Stemen ${ }^{(40)}$ comparó la reincidencia criminal entre individuos condenados por posesión de drogas (crack) que recibieron el mandato de tratamiento $(n=1494)$ vs. aquellos en libertad condicional, enviados a los servicios del tribunal, o enviados a prisión $(n=4359)$, aunque no describen el tratamiento compulsorio comunitario que recibieron los individuos. Los hallazgos sugirieron que no hubo un impacto significativo en la reincidencia criminal entre los participantes que recibieron el mandato de tratamiento en comparación con los que recibieron el mandato de libertad condicional. Es preocupante que los participantes obligados a recibir tratamiento tuvieran un riesgo significativamente mayor de reincidencia criminal en comparación con los participantes que recibieron el mandato de los servicios judiciales. Los autores concluyeron que los delincuentes obligados a tratamiento no retrocedían a una tasa menor en comparación con los delincuentes en programas alternativos.

Sun et al. ${ }^{(34)}$ compararon la recaída en el uso de crack entre una muestra de adictos en China $(n=615)$ inscriptos en detoxificación obligatoria, detoxificación voluntaria y detoxificación con reeducación a través del trabajo. La recaída global dentro de un año entre la muestra fue del $98 \% ; 22 \%$ recayeron dentro de tres días, y $52 \%$ recayeron dentro de un mes. No hubo diferencias significativas entre las tasas de recaída entre los participantes de la muestra.

Un estudio evaluó el tratamiento compulsorio de la adicción al crack en la prisión. Vaughn et al. ${ }^{(38)}$ evaluaron el programa compulsorio de tratamiento de adicciones en la prisión de Taiwan. Este programa, implementado en 1997, requería que las personas arrestadas por el uso de crack fueran sometidas a un régimen de desintoxicación de un mes después de su encarcelamiento. En ese momento, un médico determinó si los delincuentes eran dependientes de las drogas; esas personas fueron condenadas a 12 meses de prisión y a un programa de tratamiento basado en la abstinencia, incluyendo trabajo físico, asesoramiento psicológico, planificación de carrera, meditación religiosa y educación civil. Si los delincuentes no completaban satisfactoriamente el programa, eran obligados a repetirlo hasta que se complete con éxito. Una vez liberados, las personas debían pagar el costo del tratamiento. Los autores emplearon un diseño cuasi-experimental en el que las personas que realizaron el programa de tratamiento de tres meses $(n=109)$ se compararon con las personas que no se inscribieron en el programa como resultado de su encarcelamiento antes de la implementación del programa $(n=99)$ Las personas fueron entrevistadas durante la versión preliminar y después de 12 meses de la liberación de la prisión. Se usaron análisis de regresión logística multivariante para identificar cualquier diferencia significativa en el uso de drogas después del tratamiento y la reincidencia criminal. Los autores encontraron que los delincuentes inscriptos en el programa de tratamiento de drogas en las cárceles eran significativamente más propensos a participar en el uso de drogas después de la liberación y la reincidencia criminal. De esta forma, concluyeron que el sistema de tratamiento de drogas de Taiwan requiere una reforma.

Hiller et al. ${ }^{(32)}$ investigaron el impacto de una intervención compulsiva de seis meses de tratamiento de adicción residencial en la reincidencia criminal posterior al tratamiento. Los participantes en Dallas, Texas $(n=506)$ recibieron el mandato de participar en una comunidad terapéutica modificada (CT), definida como tratamiento de la adicción dentro de un entorno 
controlado dentro del cual se maximiza la supervisión. Todos los participantes fueron personas en libertad condicional o individuos arrestados por delitos relacionados con drogas en el condado de Dallas. Se compararon tres grupos: un grupo graduado $(n=290$, participantes que completaron con éxito seis meses del proceso de tratamiento de $\mathrm{CT}$ ), un grupo de abandono $(n=116$, participantes que no completaron seis meses en el CT) y un grupo de comparación $(n=100)$ compuesto de una muestra aleatoria de adictos que recayeron del condado de Dallas. Los autores compararon las tasas de encarcelamiento de 1 año y 2 años en los tres grupos de comparación, y no encontraron diferencias significativas después de 1 año en los tres grupos ( $20 \%$ del grupo que abandonó, $17 \%$ del grupo de graduados y $13 \%$ del grupo de comparación fue arrestado nuevamente y encarcelado, $\mathrm{p}>0.05)$. La proporción de participantes encarcelados dentro de 2 años no difirió significativamente entre los grupos graduados y de comparación ( $21 \%$ vs. $23 \%, p>0.05)$, aunque el grupo que abandonó tuvo una proporción significativamente mayor de participantes encarcelados en comparación con los otros dos grupos $(30 \%, \mathrm{p}<0.05)$.

Fairbairn et al. ${ }^{(36)}$ intentaron determinar si la detención en una detención compulsiva de drogas estaba asociada con el cese posterior del uso de crack entre una muestra en Bangkok $(n=422)$. Tailandia tiene un gran sistema de centros de tratamiento que busca promover la abstinencia de drogas mediante castigo, trabajo físico, la capacitación de las personas acusadas de posesión de drogas y otros delitos menores relacionados con las drogas. Generalmente, los detenidos se someten a un período de evaluación de 45 días, seguido de cuatro meses de detención y dos meses de capacitación vocacional. Los autores encontraron que el $50 \%$ de los participantes informaron un período de suspensión de al menos un año. En el análisis de regresión logística multivariada, el encarcelamiento y el tratamiento farmacológico voluntario se asociaron con el cese a largo plazo, aunque la detención compulsiva de drogas solo se asoció con el cese a corto plazo y la posterior recaída.

\section{CONCLUSIONES}

La principal paradoja que encontramos en la política de internación compulsiva es que, aunque sea una medida de privación de libertad que es configurada como violencia, esas medidas son presentadas por el poder público como soluciones de cuidado con los consumidores problemáticos de drogas. Según lo señalado por especialistas en salud mental para la internación compulsiva o involuntaria como método de recuperación de esas personas es el hecho de que un proceso de tratamiento que no es consciente y donde el consumidor no está predispuesto a su recuperación, los métodos de tratamiento difícilmente obtendrán éxito. Es importante destacar también que coaccionar a un individuo a ser protegido, contra su propia voluntad, es una medida estatal autoritaria que logra violar al mismo tiempo los inalienables derechos a la libertad ya la salud.

$\mathrm{Si}$ las medidas como la internación compulsiva se venden a través de un discurso hegemónico como un plan moderno y actual de enfrentamiento al crack, a partir de un análisis cuidadoso de lo que ha representado las políticas y acciones del gobierno federal sobre el tema, acabamos por encontrarnos la reproducción de medidas autoritarias y violentas a los derechos humanos que reproducen antiguas nociones de control biopolítico sobre vidas desnudas. El territorio de la cracolândia, como espacio público de uso de drogas por habitantes en situación de calle, también acaba por poseer sus semejanzas con la noción de campo. Ideas que, en su versión clásica como política higienista ejecutada por gobiernos autoritarios y eugenistas, son extremadamente cuestionables y reproducidos actualmente como política de gobierno. Un modelo de gestión que pretende superar una problemática tan compleja como el crack con medidas de control, que retira libertades y hiere derechos humanos, probablemente tendrá pocas probabilidades de éxito.

Con base en la literatura científica revisada por pares disponible, hay poca evidencia de que el tratamiento compulsorio con medicamentos sea efectivo para promover la abstención del consumo de drogas o para reducir la reincidencia criminal. Cabe destacar que esta revisión sistemática incluye evaluaciones no solo de los centros de detención de drogas, sino de una variedad de enfoques de tratamiento obligatorios para pacientes internados y ambulatorios. Además, las reducciones en el consumo de drogas y la reincidencia criminal como resultado de las intervenciones de internación compulsiva generalmente fueron de corto plazo o de baja significación clínica. A luz de la falta de pruebas que sugieran que el tratamiento obligatorio 
con medicamentos sea efectivo, los responsables de las políticas deberían tratar de implementar modalidades de tratamiento voluntario basadas en la evidencia para reducir los daños del consumo de drogas.

\section{Agradecimiento}

Los mejores agradecimientos al CNPq.

\section{REFERENCIAS BIBLIOGRAFICAS}

1. Dualibi LB, Ribeiro M, Laranjeira R. Profile of cocaine and crack users in Brazil. Cad Saúde Pública 2008;24(4):545-57.

2. Santos Cruz M, Andrade T, Bastos FI, Leal E, Bertoni N, Villar LM, et al. Key drug use, health and socio-economic characteristics of young crack users in two Brazilian cities. Int J Drug Policy 2013;24(5):432-38.

3. Hatsukami DK, Fischman MW. Crack cocaine and cocaine hydrochloride. Are the differences myth or reality? JAMA 1996; 276:1580-88.

4. Dunn J, Laranjeira R, Silveira DX, Formigoni MLOS, Ferri CP. Crack cocaine: an increase in use among patients attending clinics in São Paulo: 1990-1993. Subst Use Misuse 1996;31:519-27.

5. Veras $A B$. Fatores determinantes da dependência de Crack na tríplice fronteira Brasil, Bolívia e Paraguai. Interações 2016;17(1):126-133.

6. Nappo SA, Sanchez ZM, Ribeiro LA. Is there a Crack epidemic among students in Brazil?: comments on media and public health issues. Cad Saúde Pública 2012; 28(9):1643-49.

7. Peixoto $\mathrm{C}$, Prado $\mathrm{CHO}$, Rodrigues $\mathrm{CP}$, Cheda JND, Mota LBT, Veras AB. Impact of clinical and socio-demographic profiles in treatment adherence of patients attending a day hospital for alcohol and drug abuse. J Bras Psiquiatr 2010; 59(4):317-21.

8. Brasil. Lei no 10.216. Dispõe sobre a proteção e os direitos das pessoas portadoras de transtornos mentais e redireciona o modelo assistencial em saúde mental. Brasília: Diário Oficial Eletrônico; 2001.

9. Diniz ITPC, Oliveira MHB. Internação compulsória e crack: um desserviço à saúde pública. Saúde em debate 2014; 38(101): 359-67.

10. United Nations Office on drugs and crime. World Drug Report 2016. Vienna, Austria: United Nations; 2016.

11. Vos T, Barber RM, Bell B, Bertozzi-Villa A, Biryukov S; Bolliger I, et al. Global, regional, and national incidence, prevalence, and years lived with disability for 301 acute and chronic diseases and injuries in 188 countries, 1990-2013: A systematic analysis for the Global Burden of Disease Study 2013. Lancet 2015; 386(9995):743-800.
12. Dallari DA. Direitos humanos e cidadania. 2. ed. reform. São Paulo: Moderna; 2011.

13. Pêcego AJFS, Neto ZG. Crack: internação compulsória e cidadania. Revista da AJURIS 2013; 40(130):73-88.

14. Israelsson $M$, Gerdner A. Compulsory commitment to care of substance misusers: International trends during 25 years. European Addiction Res 2011; 18(6):30221.

15. WHO Regional Office for the Western Pacific. Assessment of compulsory treatment of people who use drugs in Cambodia, China, Malaysia and Viet Nam: Application of selected human rights principles. Geneva: World Health Organization; 2009.

16. Wild CT, Cunningham AJ, Ryan RM. Social pressure, coercion, and client engagement at treatment entry: a self-determination theory perspective. Addictive Behaviors 2006; 31:1858-72.

17. Bright DA, Martire KA. Does Coerced Treatment of substance-using offenders lead to improvements in substance use and recidivism? A Review of the Treatment Efficacy Literature. Australian Psychol 2012; 48(1):69-81.

18. Amarante $\mathrm{P}$, Oliveira $\mathrm{MHB}$, Torre $\mathrm{E}$, Diniz ITPC. Derechos Humanos y Salud Mental en Brasil: una historia de lucha y militancia por la justicia social y el derecho a la vida - la salud no se vende, la locura no se encierra. Rev Átopos (Salud Mental, Comunidad y Cultura) 2016; 2:1-16.

19. Hall W, Babor T, Edwards G, Laranjeira R, Marsden J, Miller $P$, et al. Compulsory detention, forced detoxification and enforced labour are not ethically acceptable or effective ways to treat addiction. Addiction 2012; 107(11), 1891-93.

20. Csete J, Pearshouse R. Detention and punishment. In the name of drug treatment. New York: Open Society Foundations; 2016.

21. Wild CT, Roberts AB, Cooper EL. Compulsory substance abuse treatment: An overview of recent findings and issues. European Addiction Res 2002; 8:84-93.

22. Stevens A, Berto D, Heckmann W, Kerschl V, Oeuvray K, van Ooyen S, et al. Quasicompulsory treatment of drug dependent offenders: An international literature 
review. Substance Use \& Misuse 2005; 40(3):269-83.

23. Robles G. Cambios y tendencias en el consumo de sustancias psicoactivas como factor de riesgo hacia la exclusión social. Zerbitzuan 2009; 45:123-34.

24. Uceda-Maza FX, Navarro-Perez JJ, PerezCosin JV. Adolescentes y drogas: su relación con la delincuencia. Revista de Estudios Sociales 2016; 58:63-75.

25. Arenari B, Dutra R. A construção social da condição de pessoa: premissas para romper o círculo vicioso de exclusão e uso problemático do Crack. In: Souza J. (Org.). Brasília: Secretaria Nacional de Política sobre Drogas, Ministério da Justiça e Cidadania; 2016.

26. Moher D, Liberati A, Tetzlaff J, Altman DG. Preferred reporting items for systematic reviews and meta-analyses: The PRISMA statement. PLoS Med 2009; 6(7): e1000097.

27. Shea BJ, Grimshaw JM, Wells GA, Boers M, Andersson N, Hamel C, et al. Development of AMSTAR: a measurement tool to assess the methodological quality of systematic reviews. BMC Med Res Methodol. 2007; 7(10):1-7.

28. Downs $S$, Black N. The feasibility of creating a checklist for the assessment of the methodological quality both of randomised and non-randomised studies of health care interventions. Journal of Epidemiol Community Health 1998; 52(6):377-384.

29. Toledo L, Cano I, Bastos L, Bertonid N, Bastos FI. Criminal justice involvement of Crack cocaine users in the city of Rio de Janeiro and Greater Metropolitan Area: Implications for public health and the public security agenda. Int Journal of Drug Policy 2017; 49(2017):65-72.

30. Heather $\mathrm{N}$. Is the concept of compulsion useful in the explanation or description of addictive behaviour and experience? Addictive Behaviors Reports 2017; 6 (2017):15-38.
31. Cruz M, Bertoni N, Bastos FI, Burnett C, Gooch J, Fisher B. Comparing key characteristics of young adult Crack users in and out-of-treatment in Rio de Janeiro, Brazil. Subst Abuse Treatment, Prevention, and Policy 2014; 9:2.

32. Hiller ML, Knight K, Simpson DD. Recidivism following mandated residential substance abuse treatment for felony probationers. The Prison Journal 2006; 86(2):230-241.

33. Strauss S, Falkin G. The first week after drug treatment: The influence of treatment on drug use among women offenders. Am J Drug and Alcohol Abuse 2001; 27(2):241264.

34. Sun B, Ye Y, Qin L. An analysis of relapse factors of 615 heroin addicts. Chinese J Drug Dependence 2001; 10(3):214-216.

35. Kelly J, Finney J, Moos R. Substance use disorder patients who are mandated to treatment: Characteristics, treatment process, and 1-and 5-year outcomes. J Subst Abuse Treatment 2005; 28(3):213223.

36. Fairbairn N, Hayashi K, Ti L, Kaplan K, Suwannawong $P$, Wood $E$, et al. Compulsory drug detention and injection drug use cessation and relapse in Bangkok, Thailand. Drug and Alcohol Rev 2015; 34(1):74-81.

37. Jansson I, Hesse M, Fridell $M$. Influence of personality disorder features on social functioning in substance-abusing women five years after compulsive residential treatment. Eur Addiction Res 2009; 15(1):25-31.

38. Vaughn $M$, Deng $F$, Lee LJ. Evaluating a prison-based drug treatment program in Taiwan. J Drug Issues 2003; 33(2):357-83.

39. Huang $K$, Zhang L, Liu J. Drug problems in contemporary China: A profile of Chinese drug users in a metropolitan area. Int J Drug Policy 2011; 22(2):128-32.

40. Rengifo AF, Stemen D. The impact of drug treatment on recidivism: Do mandatory programs make a difference? Evidence from Kansas's Senate Bill 123. Crime \& Delinquency 2010; 59(6):930-50. 